\title{
COMPATIBLE TIGHT RIESZ ORDERS
}

\author{
ANDREW WIRTH \\ (Received 22 September 1971) \\ Communicated by J. B. Miller
}

\section{Introduction}

Loy and Miller [4] have studied tight Riesz groups $(G, \leqq)$, without pseudozeros, and with $(G, \preccurlyeq)$ an $l$-group. In this paper we study abelian $l$-groups $(G, \preccurlyeq)$ on which a tight Riesz order can be defined, such that $\preccurlyeq$ is precisely the associated order. Such an order we call a CTRO (compatible tight Riesz order).

We give certain sufficient conditions for an $l$-group to have a CTRO, a useful necessary and sufficient condition, and some negative results concerning necessary conditions. The class of CTRO of a given $l$-group, ordered by set inclusion of positive cones, is directed downwards, has a maximal element, but usually is not directed upwards and has no smallest or greatest elements.

\section{Preliminaries}

Definition [5]. A tight Riesz group $(G, \leqq)$ is a partially ordered abelian group which is directed and satisfies the following interpolation property:

if $a_{1}, a_{2}, b_{1}, b_{2} \in G$ are such that

$$
a_{i}<b_{j} \text { for } i, j=1,2
$$

then there exists $c \in G$ such that

$$
a_{i}<c<b_{j} \text { for } i, j=1,2 .
$$

We write TRG to mean tight Riesz group.

Let $(G, \leqq)$ be a partially ordered abelian group. We say $x \in G$ is pseudopositive if $x \geq 0$ and $x+p>0$ for all $p>0$. We say $x$ is a pseudozero if $x$ and $-x$ are pseudopositive. If $(G, \leqq)$ has no pseudozeros we write $x>0$ to mean $x>0$ or $x$ is pseudopositive. Then $(G, \preccurlyeq)$ is a partially ordered group and we say $\preccurlyeq$ is the associated order. We note that if $a \leqslant b<c$ then $a<c$. By taking intervals $(a, b)=\{x: a<x<b\}$, where $a, b \in G a<b$, as a subbase we define the openinterval topology $\mathscr{U}$ on $G$. 
THEOREM 1 [4]. Let $(G, \leqq)$ be a TRG. Then $(G, \leqq, \mathscr{U})$ is a topological group, which is Hausdorff if and only if $(G, \leqq)$ has no pseudozeros. The family $\{(-a, a): a>0\}$ form $a$ base for $\mathscr{U}$ at $0 .(G, \leqq)$ is an antilattice (i.e. $a \wedge b$ exists if and only if $a$ and $b$ are comparable), and contains small elements (i.e. if $a>0$ and $n>0$ then there exists $b>0$ such that $a>n b>0)$. If $(G, \preccurlyeq)$ is an l-group then $\leqq$ is isolated, and $a, b>0$ implies $a \wedge b>0$.

We now present some elementary results about archimedean classes, following Loonstra's work in [3]. We assume that $(G, \preccurlyeq)$ is an abelian $l$-group.

We define an equivalence relation $\sim$ on $G^{+}=\{x: x \geqslant 0\}$. If $a, b \geqslant 0$ we write $a \sim b$ if there exist positive integers $m, n$ such that

$$
a \leqslant m b \text { and } b \leqslant n a .
$$

If $a \geqslant 0$ we write $a$ to mean the set $\{x: x \sim a\}$. The family of these archimedean classes will be denoted by $\mathscr{A}$. We write $a^{0} \preccurlyeq b^{0}$, if there exists some positive $n$ such that $a \preccurlyeq n b$. We write $a^{0} \ll b^{0}$ if $n a \preccurlyeq b$ for each positive $n$.

LEMMA 1 [3]. $\mathscr{A}, \preccurlyeq)$ is a distributive lattice with smallest element $0^{0}, a^{0} \vee b^{0}=(a \vee b)^{0}$ and $a^{0} \wedge b^{0}=(a \wedge b)^{0} .(\mathscr{A}, \lll)$ is a poset.

We say $a^{0}$ is divisible if $b / n \in G$ for each $b \in a^{0}$ and each positive $n$. We say $a^{0}$ is dense if $a^{0}=a^{0}+a^{0}$. An $l$-group $(G, \preccurlyeq)$ is said to be archimedean if $n x \preccurlyeq y$ for each integer $n$ implies that $x=0$. We denote $\mathscr{A} \backslash\left\{0^{\circ}\right\}$ by $\mathscr{A}^{*}$.

\section{Compatible tight Riesz orders}

From now on we assume that $(G, \preccurlyeq)$ is a non-trivial abelian $l$-group.

Definition. A compatible tight Riesz order (abbreviated CTRO) on $(G, \preccurlyeq)$ is a non-trivial partial order $\leqq$ making $(G, \leqq)$ a TRG without pseudozeros, and having $\preccurlyeq$ as its associated order.

LEMMA 2. Let $\leqq$ be a CTRO. Then

$$
t+x \geqslant 0 \text { for all } t>0 \text { implies that } x \geqslant 0 \text {. }
$$

Proof. Let $t>0$, then by Theorem 1 there exists $u>0$ such that $t>2 u>0$. So $t+x>2 u+x=u+(u+x) \geqslant u>0$, hence $t+x>0$ for all $t>0$, and thus by the definition of the associated order $x \geqslant 0$.

THEOREM 2. There is a one-one correspondence between CTROs on $(G, \preccurlyeq)$ and sets $T$ with the properties:

(i) $T$ is a proper dual ideal of $\left(G^{+}, \preccurlyeq\right)$

(ii) $T=T+T$

(iii) $\wedge T=0$. 
In fact the set of strictly positive elements of a CTRO satisfies conditions(i)-(iii) and vice versa.

PRoof. Suppose that $T$ is the strictly positive cone of a CTRO $\leqq$, then by Theorem $1 a, b \in T$ implies that $a \wedge b \in T$. If $a \preccurlyeq b$ and $a \in T$ then $b \in T$, also $0 \notin T$ and by definition $T$ is not empty, hence (i) follows. If $a \in T$ then there exists $b \in T$ such that $a>b>0$, by Theorem 1 . Since $a=(a-b)+b$ (ii) is satisfied. If $x \preccurlyeq t$ for all $t \in T$ then by Lemma $2-x \geqslant 0$, and so (iii) is satisfied.

Now suppose that conditions (i)-(iii) are satisfied, and write $x>0$ to mean $x \in T$. By (i) $(G, \leqq)$ is a partially ordered group. If $x, y \in G$, choose some $a>0$, then $x, y \preccurlyeq|x|+|y|<|x|+|y|+a$, so $(G, \leqq)$ is directed. If $a_{1}, a_{2}<b_{1}, b_{2}$ then

$$
b_{1} \wedge b_{2}-a_{1} \vee a_{2}=\left(b_{1}-a_{1}\right) \wedge\left(b_{1}-a_{2}\right) \wedge\left(b_{2}-a_{1}\right) \wedge\left(b_{2}-a_{2}\right)
$$

belongs to $T$, by (i). So by (ii) there exist $c, d>0$ such that $b_{1} \wedge b_{2}-a_{1} \vee a_{2}$ $=c+d$. Hence

$$
a_{1}, a_{2} \preccurlyeq a_{1} \vee a_{2}<a_{1} \vee a_{2}+c<b_{1} \wedge b_{2} \preccurlyeq b_{1}, b_{2}
$$

and so $(G, \leqq)$ is a TRG. If $x+t>0$ for all $t \in T$, then by (i) and (iii) $x \geqslant 0$. Hence $(G, \leqq)$ has no pseudozeros and $\preccurlyeq$ is its associated order.

Fuchs [1] has studied, in another context, the case where $(G, \preccurlyeq)$ is divisible.

It will prove useful to restate the above theorem in terms of archimedean classes.

THEOREM $2^{\prime}$. There is a one-one correspondence between CTROs on $(G, \preccurlyeq)$ and sets $\mathscr{T}$ with the properties:

(i) $\mathscr{T}$ is a proper dual ideal of $(\mathscr{A}, \preccurlyeq)$

(ii) if $a^{0} \in \mathscr{T}$ then there exist $b^{0}, c^{0} \in \mathscr{T}$ such that $a=b+c$

(iii) if $x^{0} \ll \mathscr{T}$ (i.e. if $x^{0} \ll y^{0}$ for all $y^{0} \in \mathscr{T}$ ) then $x=0$.

In fact the set of archimedean classes of the strictly positive elements of a CTRO satisfies conditions (i)-(iii) and vice versa.

PRoof. Suppose that $\leqq$ is a CTRO. If $a^{0}=b^{0}$ and $a>0$ then for some positive $n, n b \succcurlyeq a>0$, so by Theorem $1 b>0$. Also if $x \preccurlyeq t$ for all $t>0$, then by the existence of small elements $n x \leqslant t$ for all $t>0$ and all positive $n$. The rest of the proof follows from that of Theorem 2 .

Note. In Theorem 2' (iii) cannot be replaced by "if $x^{0} \preccurlyeq \mathscr{T}$ then $x=0$ ". For example, in $R^{2}$ with $(x, y) \geqslant 0$ if and only if $x, y \geqq 0$ let $(x, y)>0$ mean that $x>0$ and $y>0$. Then $\leqq$ is a CTRO and $\mathscr{T}=\left\{(1,1)^{\circ}\right\}$, but $(0,0)^{0} \neq(0,1)^{0}$ $\preccurlyeq(1,1)^{\circ}$.

By an abuse of language we shall call $\leqq, T, \mathscr{T}$ a CTRO as the need arises. 
THEOREM 3. Each of the following is separately a sufficient condition for $(G, \preccurlyeq)$ to posses a CTRO:

(i) $G$ is divisible

(ii) $(\mathscr{A}, \ll)$ contains a dense atom

(iii) $\left(\mathscr{A}^{*}, \ll\right)$ contains a maximal descending chain each of whose elements is dense.

Proof. Firstly we show that (iii) is sufficient. Let $\mathscr{C}$ be the chain and let $\mathscr{T}=\left\{x^{0}: x^{0} \geqslant c^{0}\right.$ for some $\left.c^{0} \in \mathscr{C}\right\}$. Then $\mathscr{T}$ satisfies conditions (i) and (iii) of Theorem 2'. If $x^{0} \in \mathscr{T}$ then for some positive $n$ and some $c^{0} \in \mathscr{C}, n x \geqslant c$. By Lemma 1 and the denseness of $c^{0}$ there exists $c_{1} \in c^{0}$ such that $c \geqslant 2 n c_{1}$. So $x \geqslant 2 c_{1}$, and $x=\left(x-c_{1}\right)+c_{1}$, hence condition (ii) of Theorem $2^{\prime}$ is satisfied.

The sufficiently of (ii) follows immediately from the above. If $G$ is divisible then (iii) is satisfied, by Hausdorff's maximal principle.

COROLlaRY 1. If $G$ is divisible and $a \succ 0$, there exists a CTRO, $\leqq$, with $a>0$.

PROOF. There exists a maximal descending chain in $\left(\mathscr{A}^{*}, \ll\right)$ through $a^{0}$; define the CTRO as in the proof above.

Corollary 2. If $(G, \preccurlyeq)$ is archimedean and divisible, then every proper dual ideal of $(\mathscr{A}, \preccurlyeq)$ is a CTRO. In particular if $a \succ 0$, then $\mathscr{T}=\left\{x^{0}: x^{0} \geqslant a^{0}\right\}$ is a CTRO.

We will show in Lemma 3 that there exists an archimedean $l$-group with a CTRO, which does not have any dense archimedean classes, except for $0^{\circ}$.

Let $\left\{\left(F_{i}, \preccurlyeq\right): i \in I\right\}$ be a non-empty family of fully ordered non-trivial abelian groups. We denote the full direct product by $\Pi F_{i}$ and the direct sum by $\Sigma F_{i}$. If $x \in \Pi F_{i}$ we write its $i$ th component as $x(i)$ and we write $x \geqslant 0$ to mean $x(i) \geqslant 0$ for all $i$. We call this the pointwise order.

THEOREM 4. The l-group $\left(\Pi F_{i}, \preccurlyeq\right)$ has a CTRO if and only if either $I$ is infinite or at least one $\left(F_{i}, \preccurlyeq\right)$ is dense. The l-group $\left(\Sigma F_{i}, \preccurlyeq\right)$ has a CTRO if and only if at least one $\left(F_{i}, \preccurlyeq\right)$ is dense.

Proof. Suppose $I$ is infinite, then select a countably infinite subset $J \subseteq I$, and write $J=\{1,2,3, \cdots\}$. Choose any $k_{n} \succ 0 \quad k_{n} \in F_{n}$, and if $x \in \Pi F_{i}$, write $x>0$ to mean that $x>0$ and there exist positive integers $m_{n}$ such that $x(n) \succcurlyeq m_{n} k_{n}$ and $\lim _{n} m_{n}=\infty$. Then $\leqq$ is a CTRO on $\left(\Pi F_{i}, \preccurlyeq\right)$, by Theorem 2 .

Suppose $\left(F_{i_{0}}, \preccurlyeq\right)$ is dense and for $x \in \Pi F_{i}$ write $x>0$ to mean that $x>0$ and $x\left(i_{0}\right) \succ 0$. Then $\leqq$ is a CTRO on $\left(\Pi F_{i}, \preccurlyeq\right)$.

Suppose that $I$ is finite, none of the $\left(F_{i}, \preccurlyeq\right)$ are dense, and $\leqq$ is a CTRO on 
$\left(\Pi F_{i}, \preccurlyeq\right)$. There exist atoms $a_{i}$ in $\left(F_{i}^{+}, \preccurlyeq\right)$. Suppose that for each $i$ there exists $x_{i}>0$ with $x_{i}(i)^{0} \preccurlyeq a_{i}^{0}$. Then by the existence of small elements there exist $y_{i}>0$ with $y_{i}(i)=0$. So $\wedge y_{i}=0$, but by Theorem $1 \wedge y_{i}>0$. So in fact there exists $i_{0}$ such that, if $x>0$ then $x\left(i_{0}\right) \gg a_{i_{0}}$. Now define $q>0$ by, $q(i)=0$ if $i \neq i_{0}$ and $q\left(i_{0}\right)=a_{i_{0}}$. Then $q$ is a pseudozero, and so $\leqq$ is not a CTRO.

The result for $\Sigma F_{i}$ follows easily from the above.

COROLLARY. A fully ordered group $(G, \preccurlyeq)$ has either no CTRO, or is dense and has exactly one CTRO, $\preccurlyeq$ itself.

LEMMA 3. There exists an archimedean l-group with a CTRO but without a dense archimedean class, save $0^{\circ}$. There exists an l-group with the set $\left\{x^{0}: x^{0} \geqslant a^{0}\right\}$ a CTRO but $a^{0}$ not divisible.

Proof. Let $(G, \preccurlyeq)$ be the l-group of all sequences of integers with pointwise order. By Theorem $4(G, \preccurlyeq)$ has a CTRO. If $a>0$ and $a(i)>0$, say, let $b$ be defined by $b(j)=a(j)$ if $j \neq i$, and $b(i)=1$. Then $b \in a^{0}$, but clearly if $b=c+d$ then either $c \notin b^{0}$ or $d \notin b^{0}$.

Let $(G, \preccurlyeq)$ be the subgroup of $R$, with the usual order, generated by $\left\{\frac{1}{3}, \frac{1}{5}, \frac{1}{7}, \cdots\right\}$. By Theorem 4 Corollary $(G, \preccurlyeq)$ has a CTRO but $\frac{1}{2} \cdot \frac{1}{3}$ does not belong to $G$.

THEOREM 5. If $\mathscr{T}$ is a CTRO with open-interval topology $\mathscr{U}$, then the following are equivalent:

(i) $(G, \mathscr{U})$ is first countable.

(ii) There exists a countable descending chain $a_{1}^{0} \succcurlyeq a_{2}^{0} \succcurlyeq \cdots$ in $(\mathscr{T}, \preccurlyeq)$ with $\mathscr{T}=\left\{x^{0}: x^{0} \geqslant a_{n}^{0}\right.$ for some $\left.n\right\}$.

(iii) $(G, \mathscr{U})$ has a translation-invariant metric, $\rho$, satisfying:

$$
|x| \preccurlyeq|y| \text { implies } \rho(x, 0) \leqq \rho(y, 0) .
$$

Proof. (i) implies (ii). If $(G, \mathscr{U})$ is first countable then by Theorem 1 there exists a sequence $\left\{p_{n}\right\}$ such that, if $x>0$ then $x>p_{n}$ for some $n$, and $p_{n}>0$ for all $n$. Let $a_{n}=p_{1} \wedge \cdots \wedge p_{n}$, then $a_{1} \succcurlyeq a_{2} \succcurlyeq \cdots>0$, and $\mathscr{T}=\left\{x^{0}: x^{0} \succcurlyeq a_{n}^{0}\right.$ for some $n$ \}.

(ii) implies (i). Suppose $\mathscr{T}=\left\{x^{0}: x^{0} \succcurlyeq a_{n}^{0}\right.$ for some $\left.n\right\}$ and $a_{1}^{0} \succcurlyeq a_{2}^{0} \succcurlyeq \cdots$, then by Theorem 1 , there exist $p_{i n}>0$, such that $a_{i}>n p_{i n}>0$. If $x>0$, then there exist integers $i$ and $n$ such that $n x \geqslant a_{i}$, so $x>p_{i n}$.

(i) implies (iii). If $|x|^{0} \preccurlyeq p_{r}^{0}$ define $p_{r}(x)$ by

$$
p_{r}(x)=\inf \left\{\frac{m}{n}:-m p_{r} \preccurlyeq n x \preccurlyeq m p_{r}, m \geqq 0, n>0\right\} .
$$


Otherwise write $p_{r}(x)=\infty$. Now let

$$
\rho(x, y)=\sum_{n=1}^{\infty} \min \left\{p_{n}(x-y), \frac{1}{2^{n}}\right\},
$$

then $\rho$ is the required metric.

Any Hausdorff abelian topological group which is first countable has a translation-invariant metric [2].

Note. It can be shown that in a Banach lattice with strong unit the metric, corresponding to the CTRO consisting precisely of the strong units, is equivalent to the original metric.

\section{The class of CTROs}

Let $(G, \preccurlyeq)$ be a non-trivial abelian $l$-group with a CTRO. Denote the family of CTROs by $\left\{R_{\alpha}\right\}$ and partially order it by set inclusion (of the strictly positive cones).

THEOREM 6. The poset $\left\{R_{\alpha}, \subseteq\right\}$ is directed downwards. If $G$ is divisible then $\left\{R_{\alpha}, \subseteq\right\}$ is directed upwards if and only if $(G, \preccurlyeq)$ is fully ordered; and $T_{1} \wedge T_{2}=T_{1} \cap T_{2}$.

PROoF. Suppose that $T_{1}$ and $T_{2}$ are CTROs, and let $T_{3}=T_{1}+T_{2}=\left\{t_{3}: t_{3}\right.$ $\left.=t_{1}+t_{2}, t_{1} \in T_{1}, t_{2} \in T_{2}\right\}$. If $x \geqslant t_{1}+t_{2}$, with $t_{1} \in T_{1}, t_{2} \in T_{2}$, then $x-t_{1} \succcurlyeq t_{2}$, so $x-t_{1} \in T_{2}$ and $x \in T_{3}$. If $t_{1}, u_{1} \in T_{1}$ and $t_{2}, u_{2} \in T_{2}$ then $\left(t_{1}+t_{2}\right) \wedge\left(u_{1}+u_{2}\right)$ $\succcurlyeq t_{1} \wedge u_{1}+t_{2} \wedge u_{2}$. So $T_{3}$ satisfies condition (i) of Theorem 2 , and also condition (ii). If $t_{1}+t_{2} \geqslant x$ for all $t_{1} \in T_{1}, t_{2} \in T_{2}$, then $0 \preccurlyeq x-t_{1}$ for all $t_{1} \in T_{1}$, since $\wedge T_{2}=0$. So in fact $x \preccurlyeq 0$, since $\wedge T_{1}=0$. Hence $T_{3}$ satisfies condition (iii) of Theorem 2, and clearly $T_{3} \subseteq T_{1}, T_{2}$.

Suppose that $(G, \preccurlyeq)$ is divisible and not fully ordered. Then there exist $a_{1}, a_{2} \succ 0$ such that $a_{1} \wedge a_{2}=0$. By Theorem 3 Corollary 1 there exist CTROs $T_{1}, T_{2}$ with $a_{1} \in T_{1}, a_{2} \in a_{2}$. If there exists a CTRO $T_{0}$ such that $T_{0} \supseteq T_{1}, T_{2}$ then $0=a_{1} \wedge a_{2} \in T_{0}$, by Theorem 1. Hence $\left\{R_{\alpha}, \subseteq\right\}$ is not directed upwards.

If $(G, \preccurlyeq)$ is divisible and fully ordered, then by Theorem 4 Corollary $\left\{R_{a}\right\}$ is a singleton.

Suppose that $G$ is divisible and $T_{1}, T_{2}$ are CTROs. Then $T_{1} \cap T_{2}$ satisfies conditions (i) and (ii) of Theorem 2. Since $T_{1}+T_{2} \subseteq T_{1} \cap T_{2}$, hence by the above $T_{1} \cap T_{2}$ satisfies condition (iii) of Theorem 2 .

Note. The fact that $\left\{R_{\alpha}, \subseteq\right\}$ is directed downwards will be used in [6] paper to define a convergence (in terms of the order) on any abelian partially ordered group, which agrees with relative uniform convergence on directed integrally closed partially ordered vector spaces, and with order convergence on fully ordered abelian groups. 
Lemma 4. Let $(G, \preccurlyeq)$ be divisible and archimedean, then it has a smallest CTRO if and only if $(G, \preccurlyeq)$ has a strong unit. In fact the smallest CTRO consists precisely of the strong units.

Proof. Suppose that $a$ belongs to the smallest CTRO. Then by Theorem 3 Corollary $2 a^{0} \geqslant x^{0}$ for all $x>0$. Hence $a$ is a strong unit and the rest follows easily.

The $l$-group $R \times Z$ (pointwise order) is archimedean and has exactly one CTRO, containing elements other than strong units. The group of real-valued sequences with finitely many non-zero terms can be fully ordered, by letting its strictly positive cone consist of sequences whose last non-zero term is positive. By Theorem 4 Corollary this group has exacly one CTRO, but does not contain any strong units.

Lemma 5. The poset $\left\{R_{\alpha}, \subseteq\right\}$ has a maximal element.

Proof. The standard Zorn's Lemma argument proves the existence of a maximal element.

\section{Acknowledgments}

The author would like to thank Professor J. B. Miller and Dr B. F. Sherman for suggesting improvements to the paper. This research was carried out while the author held a Commonwealth Postgraduate Award.

\section{References}

[1] L. Fuchs 'Approximation of lattice-ordered groups', Annales Univ. Sci. Budapest, Sect. Math. 8 (1965), 187-203.

[2] E. Hewitt and K. A. Ross Abstract harmonic analysis I (Springer, Berlin, 1963), 70.

[3] F. Loonstra 'The classes of partially ordered groups', Compositio Math. 9 (1951), 130-140.

[4] R. J. Loy and J. B. Miller 'Tight Riesz groups' J. Aust. Math. Soc., 13 (1972), 224-240.

[5] J. B. Miller 'Higher derivations on Banach algebras', Amer. J. Math. 92 (1970), 301-331.

[6] A. Wirth 'Convergence in partially ordered groups', Proc. Edin. Math. Soc.. to appear.

Department of Mathematics

Monash University

Victoria, 3168

Australia 